\title{
PAKRIMPUNGANG SANJAK MANGKASARAK BORIK MALABBIRITTA
}

\section{Penulis}

Prof.Dr.Hj. Kembong Daeng,M.Hum.

Penerbit CV. Cahaya Bintang Cemerlang 


\title{
PAKRIMPUNGANG SANJAK MANGKASARAK \\ BORIK MALABBIRITTA \\ Penulis:
}

Prof.Dr.Hj. Kembong Daeng, M.Hum.

ISBN 978-623-6032-13-8

\author{
Editor : \\ Dr. Hajrah, S.S.,M.Pd \\ Penyunting: \\ Muh Yunus Nabbi \\ Desain Sampul dan Tata Letak \\ Muh. Yunus Nabbi \\ Penerbit: \\ Penerbit CV. CAHAYA BINTANG CEMERLANG \\ Redaksi :
}

Jl. Dr. Wahidin Sudirohusodo BTN Indira Residence Blok E No.

10

Sungguminasa Kab. Gowa

No. HP: 085256649684

Email : muhyunusnabbi@gmail.com

Distributor Tunggal

Penerbit CV. CAHAYA BINTANG CEMERLANG

Jl. Dr. Wahidin Sudirohusodo BTN Indira Residence Blok E No. 10

Sungguminasa Kab. Gowa

No. HP: $081937538693 /$ WA: 085290480054

http//cv-cahayabintangcemerlang.co.id

Anggota UMKM Nomor : 04933-0615-20

Anggota IKAPI Nomor : 027/SSL/2020

Cetakan Pertama, Januari 2022

Hak cipta dilindungi Undang-undang

Dilarang memperbanyak karya tulis ini dalam bentuk dan dengan cara Apapun tanpa ijin tertulis dari Penerbit. 


\section{ALAS KATA DAN APRESIASI KEPADA PENULIS \\ Dr. Hj. Ery Iswari, M.Hum.}

Ucapan puji syukur ke hadirat Allah Swt selalu kami panjatkan karena dengan izin-Nya sehingga buku dari Kembong Daeng yang berjudul "Pakrimpungang Sanjak Mangkasarak Bori Malabbiritta" dapat diterbitkan oleh Penerbit CV.Cahaya Bintang Cemerlang. Buku ini berisi 66 buah sajak berbahasa Makassar yang merupakan hasil coretan pena Penulis dan merupakan ekspresi buah pikiran serta ungkapan rasa berdasarkan pengalaman lahir batin sehingga beberapa sajak sangat terasa menyentuh hati.

Buku yang berisi sajak berbahasa Makassar ini dapat menjadi sumber rujukan Bahasa \& Sastra Makassar, khususnya kajian sastra bagi anak didik mulai tingkat SD,SMP,SMA, hingga Perguruan Tinggi karena pilihan leksikal yang digunakan merupakan kosakata seharihari. Penggunaan kosakata yang sederhana dapat mempermudah pembaca untuk lebih menghayati makna yang terkandung di dalamnya sehingga tidak menguras pikiran dan membutuhkan interpretasi yang tinggi untuk memperoleh pesan yang terkandung dalam setiap bait-bait sajak.

Sajak-sajak dalam buku ini juga dapat dijadikan referensi untuk mengajarkan anak didik atau mahasiswa tentang karakter positif yang berbasis dari kearifan lokal dan filosfi hidup orang Makassar. 
Judul buku ini mengadopsi judul sajak yang pertama yaitu "Borik Malabbiritta" di mana isinya tampak seperti ada dialog antara kampung (borik) yang satu dengan yang lainnya, dan saling mengkritisi serta saling mengingatkan karena kealpaan manusia yang mengisi ruangruang kehidupan di dunia. Borik Malabbiritta saja ikut khawatir tentang kondisi kehidupan saat ini yang mendeskripsikan manusia seperti tidak lagi terlalu peduli dengan filosofi hidup orang Makassar sehingga membuatnya gusar. Si Borik menghimbau kita semua untuk tetap menghadirkan siri na pace dalam bersosialisasi dan menempatkan adat pada posisi yang semestinya agar kampung kita dapat tetap sejahtera dan damai.

Hasil internalisasi olah rasa dan pengalaman batin Penulis dari berbagai dimensi, mulai dari kehidupan keluarga, harapan-harapan, nasihat tentang kehidupan, rasa apresiasi, hingga pentingnya pendidikan semua diramu dalam buku ini; sehingga tampak seperti sajian sajak yang paripurna dari aspek penghayatan menjalani kehidupan dengan berbagai dinamika. Sajak-sajak Makassar dalam buku ini dapat kita simak bukan hanya dari dimensi isi secara keseluruhan, tapi kita juga dapat belajar kembali menyelami penggunaan diksi yang mungkin saja tidak lagi pernah kita dengar dalam keseharian kita. Hal ini terindikasi pada beberapa penggunaan kata dan rangkaian kata yang konotatif dengan menggunakan metafora atau analogi dari beberapa larik sajak yang membuat untaian katanya terasa lebih puitis. 
Saya atas nama pribadi mengucapkan selamat dan menyatakan apresiasi yang tinggi kepada Penulis atas terbitnya buku kumpulan sajak berbahasa Makassar ini. Buku kumpulan sajak yang berbahasa Makassar masih sangat langka. Oleh karena itu, diharapkan buku ini dapat menginspirasi banyak orang, baik dari kalangan pendidik, pemerhati bahasa dan budaya lokal, maupun profesi lainnya agar dapat tetap menjaga eksistensi bahasa dan sastra lokal dalam berbagai bentuknya. Semoga berkah Allah Swt senantiasa menyertai kita semua.

Salamakki....

Sungguminasa, Desember 2021

Dr.Hj.Ery Iswary,M.Hum 


\section{KANA PAPPAKARIOLO}

Kupakarioloi pappalak sukkurukku mange ri Karaeng Allahu Taala nasabak Nasareku buku magassing, pappijojjok, siagang panngamaseang sanggenna akkulle kupakrappungang anne sanjak Mangkasarak bate limangku nakkulle ajjari sekre bobbok. Sanjak Mangkasarak bate ukirikku sangkamma sekre pappidalek Nasareangak Karaeng Allahu Taala.

Kanniakkanna anne bobboka sannak nikarannuanna nasabak kurannna injapa sanjak-sanjak Mangkassarak makaakkulle nibaca iareka nipappilajari ri mahasiswayya kammayya tompa anak-anak pasikolayya. Jari, kuminasai kaniakkanna anne bobboka barang ajjari bobbok makaallulle niparek bacang mabajik nakkulle anngerang matu-matu, bajik na ri doseng ampanngajjarangi Puisi Mangkasaraka, guru-gurua, mahasiswayya, siagang pasikolayya.

Anne sanjak-sanjakku akkullei nibassukang lanri niakna pallompoi sumangak battu ri alasiapayya tumalabbirik, kammayyami Rektor Univeritas Negeri Makassar, Dekan Fakultas Bahasa dan Sastra, Ketua Jurusan Bahasa dan Sastra Indonesia, siagang sikamma doseng niaka anngajarak ri Program Studi Pendidikan Bahasa dan Sastra Daerah. Lanri kammannami anjo kuppabattu tarima kasi majai dudu. Dasi na dasi barang Nabalasajji pakmaik bajitta Karaeng Allahu Taala. Kupabattui pole tarima kasi assangkammayya mange ri tumalabbiritta 
Dr.Ery Iswari, M.Hum na Dr. Hajrah, S.S.,M.Pd. lanri eroknaja assare kana sambutan na pappakabaji ri kaniakkanna anne bobboka.

Taena todong kutakkaluppa appabattu tarima kasi majai dudu mange ri Daengku Dr.H. Muh.Yahya, M.Pd. siagang anakanak tuningaiku Nurul Fajriah yahya, S.Psi., Syahratul Hawaisa Yahya, S.Farm., Nur Fajar Malik, S.Tr.Pi. (mintu), dan Muhammad Fahmi Yahya lanri laccukna nyawana salleang akkulle kupaknassa anne sanjaka ajjari bobbok. Dasi na dasi barang anne sanjak bate limangku nakkulle anggappa panngamaseang battu ri Karaeng Allahu Taala na tuli massolong mange ruwayya tunnganakkangku.

Taena rupatau sukkuk pammuntulukna, kamma tonji anne ukirang bate limangku maknassami jai kakuranganna bajik na ri bukuang kananna kammayya tompa ukiranna. Jari kupalaki pakmaij bajitta ri katte tau ambacayyai sollanna na kipakakanang punna niak kana majule na kipakanaba punna niak ukirang masala.Dasi na dasi barang Nabalasajji Karaeng Allahu Taala sikamma pakmaik bajitta. Barangkammai apa nanggeranja matumatu na pallompoi sumangak ri katte tau ambacayaai anne sanjak-sanjak Mangkasaraka.

Makassar, 1 Desember 2021

Prof.Dr.Hj.Kembong Daeng,M.Hum. 


\section{PRAKATA}

Puji dan syukur penulis senantiasa haturkan kepada Allah Swt. Tuhan Maha Pencipta karena berkat rahmat dan hidayahNyalah sehingga penulis dapat mempersembahkan kumpulan puisi Makassar yang berjudul Pakrimpungang Sanjak Mangkasarak "Borik Malabbiritta" dengan baik.

Sanjak Mangkasarak merupakan karya sastra yang masih langka sehingga kehadirannya sangat dibutuhkan baik oleh dosen, guru, mahasiswa, siswa, maupun masyarakat umum. Dengan demikian, kehadiran buku sastra ini dapat dijadikan sebagai referensi yang dapat memperkaya pengetahuan pembaca tentang sastra Makassar, khususnya karya sastra yang berbentuk puisi bebas. Puisi ini diberi judul Pakrimpungang Sanjak Makassarak karena menghimpun kumpulan puisi bebas dalam bahasa Makassar yang merupakan kasya asli penulis. Selanjtnya, "Borik Malabbiritta" merupakan salah satu judul puisi bebas yang pertama dikarang oleh penulis sehingga judul ini dijadikan sebagai judul buku.

Karya ini dapat diterbitkan dalam bentuk buku berkat adanya dukungan dan motivasi berkarya dan berinovasi dari Rektor Universita Negeri Makassar, Dekan Fakultas Bahasa dan Sastra, Ketua Jurusan Bahasa dan Sastra Indonesia, dan dosen Pendidikan Bahasa dan Sastra Daerah. Oleh karena itu, penulis menyampaikan terima kasih yang setulus-tulusnya. Terima kasih 
yang sama, penulis tujuakan kepada Dr. Salam, M.Pd. yang telah memberikan sambutan dan Dr. Hajrah, S.S., M.Pd. sebagai editor dalam penerbitan buku ini. Terima kasih pula kepada penerbit yang bersedia menerbitkan karya penulis.

Terima kasih yang tulus juga disampaikan kepada suami tercinta Dr.H.Muh. Yahya, M.Pd. dan anak-anak tersayang Nurul Fajriah yahya, S.Psi., Syahratul Hawaisa Yahya, S.Farm., Nur Fajar Malik, S.Tr.Pi. (menantu), dan Muhammad Fahmi Yahya atas ketulusan dan keihlasannya sehingga penulis dapat melahirkan sanjak-sanjak Mangkasarak. Semoga karya ini menjadi amal jariah yang selalu mengalir kepada kedua orang tua tercinta.

Penulis sebagai manusia yang tidak sempurna memiliki kekurangan dan keterbatasan, demikian juga halnya dengan karya ini masih memiliki kesalahan baik dari segi diksi maupun ejaan. Untuk itu, diharapkan kiranya pembaca memperbaiki kesalahan yang ditemukan dan mengapresiasi dengan baik sanjak demi sanjak semoga dapat memberikan manfaat bagi pembaca agar termotivasi untuk membaca sastra Makassar. Akhirnya, penulis berdoa semoga Allah Swt. memberikan pahala yang setimpal kepada semua pihak yang memberikan andil dalam penerbitan buku ini. Selamat mengapresiasi sanjak-sanjak Mangkasarak!

Makassar,1 Desember 2021

Prof.Dr.Hj.Kembong Daeng,M.Hum. 


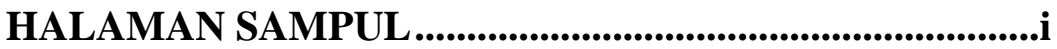

HALAMAN REDAKSI PENERBIT ...........................................ii

ALASKATA DAN APRESIASI KEPADA PENULIS .........iii

KANA PAPPAKARIOLO ..................................................vi

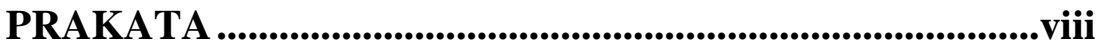

DAFTAR ISI...............................................................................................

(1) Borik Malabbiritta..........................................................1

(2) Passolongan Cerakku ...............................................................2

(3) Takalarak Butta Panrannuangku ......................................4

(4) Mangkasarak Borik Masunggu ...........................................6

(5) Pappasanna Tutowaku ...........................................................8

(6) Anak Manngalleanga Kana ...................................................10

(7) Karaeng Malompoa ...................................................................11

(8) Ammakku Anrong Kalengku...............................................13

(9) Kuparabbami Ulungku ..........................................................14

(10) Anrong Manggeku ................................................................16

(11) Kurapang Bulaeng Tiknok ................................................17

(12) Kereji Janjinta .........................................................................19

(13) Manggeku ..........................................................................21

(14) Kaddek Assikola Tonjak....................................................22

(15) Kaddek Tena Kussikola ......................................................24

(16) Teaki Makring Najinak.......................................................25 
(17) Pikkiri la Kikananga ............................................................27

(18) Maeki Sipitangarri ...........................................................28

(19) Pacce ta Naebbak Lading...................................................29

(20) Ladingku Tonji Kupake........................................................31

(21) Pakabajiki Jamanta................................................................32

(22) Teaki Pacalla-calla....................................................................33

(23) Kaddejji Kapang Kiasseng ...............................................35

(24) Kisailei Bokota ........................................................................36

(25) Kucinitta Anngarrukku ...........................................................38

(26) Kicinikku Anngarutta ........................................................39

(27) Erokki Tea......................................................................41

(28) Ningai ta Nijampangi ............................................................42

(29) Tekne ta Nigollai ...........................................................44

(30) Janna ta Nisantanngi ............................................................45

(31) Late Bunga Ta Malate Cinikanna...................................47

(32) Jammeng ta Nikaluppai ......................................................48

(33) Bija Pammanakangku ......................................................49

(34) Teaki Lanre Mattayang ........................................................51

(35) Kupabellami ri Ati............................................................52

(36) Bokomak ri Panngainta .......................................................53

(37) Anakku ......................................................................................55

(38) Pakminasa ri Anakku..........................................................56

(39) Kupalilimi Kalengku ................................................................58

(40) Kutetemminne Simpungku ...............................................59 
(41) Kulewai Nyawaku.........................................................60

(42) Kusingarri Paccinikku ..................................................61

(43) Panngamaseanna Bijangku ...............................................62

(44) Kapeang Tommak ...............................................................63

(45) Ammattik Jeknek Matangku.............................................64

(46) Niak Tonja Antu Sallang ....................................................66

(47) Siapaminne Salloku ...............................................................67

(48) Manna Jannammo Tinroku..............................................68

(49) Carammengi Kalenta .......................................................69

(50) Sipakatau ...................................................................................71

(51) Teaki Takkaluppai .............................................................72

(52) Kilambusi Paknyawanta .......................................................73

(53) Curak Lipak Napilei.....................................................75

(54) Teaki Ranggaselai....................................................................76

(55) Pakkeke Mappasisaklak....................................................77

(56) Teaki Lanre Mattayang ......................................................79

(57) Tojengangi Kananta ............................................................81

(58) Danngalakminjo Ulunna .....................................................82

(59) Jeknek Matangku .................................................................84

(60) Rannungku ri Dosengku

(Ibu Barbara Friberg/Timoty) .........................................86

(61) Pappakalabbirikku ri Gurungku ...................................88

(62) Ruwayya Tunnganakkangku.............................................90

(63) Allerang Jeknek Matangku ............................................91 
(64) Julu Borikku .....................................................................93

(65) Singai Sikanakkuki............................................................95

(66) Tea Tonjak Nitunai ........................................................97 


\section{(1) Borik Malabbiritta}

Anne kamma borik malabbiritta

Niakmi i lalang simpung pakmaik

Dodong nyawana

Tuttuluk buku-bukunna

Akkutaknammi borik maraenga

E ... Saribbattang

Anngapa nukamma dudu?

Riolo ... i kau nikana-kana

Borik malabbirik, borik masunggu,

amang pakrasangannu,

salewangang tumakbuttanu

Mingka, anne kamma?

Appiwalimi borik malabbiritta

Iyek ... Saribbattang

Kamma tojengintu pakkananta

Riolo ... tumakbuttaku

Erok inji sipakatau, sipakalabbirik,

sipakaingak punna niak takkaluppa

Mingka ..., anne kamma

tumakbuttaku sipakgadang 
Doekamami naparek saribbattang

Barang-barangamami naparek pannyaleori

Anaka tanngasseng anrupa tau towa

Tau towayya takkulle

nitakgalak ulu kananna

Tassungekngekmi borik malabbiritta

Nakkana

E ... tumakbuttaku

Pakniakkangak sirikku na pacceku

Sungkeangak bongongku

Maemaki ajjulu kana, abbulo sibatang,

accerak sitongka-tongka

Paempoi adaka ri memanganna

Na nibangung borik malabbiritta

Barang kammai apa

nammoterejja salewangang

Kamma ri memanganna

Katangka (Gowa), 2002 


\section{(2) Passolongang Cerakku}

Bontolebang butta kalassukangku

Butta passolongang cerakku

Tampak pakrimpunganna

Bija pammanakangku

Bontolebanna Moncongkomba

Gallarranna gassing gauk

Ajjari sabbi ri anak cucunta

Kalabbiranna tumakbuttana

Akkulle nialle picuru

Lompo pacce na lompo sirik

Bajik adak na bajik ampe

Ri sikamma tumabbuttayya

Paentengi kalambusanga na kontu tojenga

Ajaraki anak cucunta akgauk mabajik

Malliliang parek kodi

Rannuangi pammarentata

i katte tumakbuttayya

sipakaingak punna ammotere ri kalambusang

Anngarimananngiki ri tabbalatta

i katte pammarentayya

Nampapi antu salewangang tumabbuttata 
Niajjantu kullenna na amang

salewangang pakrasanganta

Ukrangi pappasanna

tutowa malabbiritta

Assamaturukki kibbulo sibatang

i katte sipammanakang

Na kipattaena kodi keroa

na lompo kella-kellayya

Gauk bajik nilamung gauk bajik nitaroi,

Kodi nilamung kodi nirampe-rampe

Kikarannuangi na kisukkuri pappidallek-Na

Karaeng Allahu Taala

Upakki ri lino kiupak todong allo ri boko

Makassar, 25 Januari 2016 


\section{(3) Takalarak Butta Panrannuangku}

Takalarak borik masunggu

Akminasa tuli tekne, tuli empo salewangang

Kitea sigenra-genra

Assamaturukki manggaukang parek bajik

Liliangi sikontu parek kodia

Anaka manngalleangki kana tutowa,

tutowayya ajjariki picuru ri anak cucunta

Rannuki sikarannuang, pacceki sipappaccei

Anngukrangi tamattappuk

Ri Karaeng Allahu Taala

Borik malabbiritta, appasang ri jari-jarina

Ukrangi pappasanna tutowa malakbairitta

Tutu ri kana-kana, ingak ri panggaukang

Tantang ri kontu tojeng

Amminawang ri tubarani, manngasseng, malambusuk pakmaik

Pakniaki siritta na pacceta

Ammentengki ri katojengang

Nyawata teaki sakgai punna sirik la tabballe

Rannuangi tau akkullea nipatappak 
Apparekki ampe-ampe mabajik

$\mathrm{Na}$ narampe ampe bajitta taua

Niak antu sallang na nipelei

lamungang la mattimboa

Ulu kanannajintu taua nitakgalak

Akkaresoki na kitunulu

Na kitambung ri erok Panngamaseang-Na

Gassingi kalenta, teaki eroki tuli nipatiru

Kakkaresopakintu nakkulle tappainra tallasatta

Ukrangi Karaeng Mappajjaria tamattappuk ri atinta!

10 Februari 2016 


\section{(4) Mangkasarak Borik Masunggu}

Mangkasarak pakrasanganta

Butta passolongang ceratta

Anrinimakinne nilassukang

Nitaranak, nipakalompo, nipasikola

Ri anrong mangge tullassukanta

Mangkasarak borik masunggu

Coppong buttana

Salewangang tumabbuttana

Akrupa-rupa adak na panngadakkanna

Manna na kamma

Tuliji sipakatau, sipakalabbirik, sipakaingak,

situlung sikamaseang

Ri julu pakrasanganna

Mangkasarak borik masomberek

Bajik pakmaik, mabajik adak

sikontu tumakbuttana

Pammarentana manngai ri tabbalakna

Rakyaka massamaturuki na erok majjulu kana

Tau towayya naassengi kalenna tau towa

Anaka naassengi kalenna anak

Tupanritayya tena nalanre anngajarak 
Tukalumannyanga mannabai assare-sare

Anrinimakinne ri butta malabbiritta

Niajarak singai sikamaseang

Sipassiriki sipappaccei

Mangkasarak borik tantang ri kontu tojeng

Tumakbuttana ia kana ia gauk

Toddoppuli ri panggappa

Mammenteng ri kalambusang

Mabajik adak mammempo ri panngadakkang

I katte sipammanakang

E ... sikamma saribbattangku

Teaki makring napaklingu tumaraeng

Teaki pitigiok-gioki

pitipinak-pinawanngi

Ri tukgauk tannaba-naba

Maeki assamaturuk, abbulosibatang

Na nibangung pakrasanganta

Dasi na dasi nakkulle niparek picuru

Ri pakrasangang maraenganga

Makassar, 4 April 2018 
(5) Pappasanna Tutowaku

Tabek, pammopporammamak

Ri dallekang malabbiritta

Erokak anne ambacangki

Puisi "Pappasanna Tutowaku"

I nakkeminne jarina tubarania

Tau manngalleanga

kana panngajarak

Malliliang pappisangka

Manggaukang parek bajik

Pappasanna tutowaku

Manna kalewang takkalang ri dallekangnu

Badik tattinting ri tanngana empoannu

Poke tattinrak ri sakrinna onjokannu

Teako mallak punna tenaja pusala

Teako monoki punna tojeng nupajappa

Teako biluki punna lambusuk nuolo

Teako makring naurikang tau sala

Kalamanngannumo puli

I lalang pabbundukannu

Kuntungannumo tallang 
I lalang passimombalannu

Alangakannayya lari niondang berang

$\mathrm{Na}$ taena kasalannu

Satuli-tuli kananna tutowaku

Pappasanna ri anak jari tauna

Ammentengko ri kalambusang

Tantang ri kontu tojeng

Numanngalleang tangarak ri tupanrita

Numminawang ri tulambusuk nyawana

Teako makring annganre ta siratannu

Anngayao na teyai kau pata

Dallenu tommo sukkuri

Na masannang pakmaiknu

Salewangang tallasaknu

Punna bajijja gioknu

Lambusuk jappa-jappanu

Tantang ri kontu tojeng

Manngai manngamaseang

Ri parannu rupa tau

Nuerok tonja nipaingak

ri tau malabbiriknu

Allemi sekre-sekrea 
Nutanjeng ri Alla Taala

Tenamo antu na nusassalak kalennu

Tallasak empo ri lino

Najariang tubarani

Makassar, 3 April 


\section{(6) Anak Manngalleanga Kana}

Papppasanna tutowa malabbirikku

Ri kambe jari-jarina

Kuparek pakkallik batangkale

Kuparek picuru ri giok-giokku

Kuparek sikkok majarrek

Ri sikontu tappuk kanangku

Pallompoi sumangak

I lalang tallasakku

Kujjari tau mabajik

Manngalleang kana pannganjarak

Malliliang pappisangka

Nampak i lalang battang

$\mathrm{Na}$ nakminasa tutowaku

Lassuk ri lino

Ajjari anak masunggu

Narannuang tau jai

Anak-anak, tau towa

Tau lolo, tau rungka

Manngai marannu aseng

Lanri tena kulebbak appssalasa

I nakkeminne

Anak manngalleanga kana 
Tantang ri kontu tojeng

Lambusuk ri panggaukang

Malompo sirik

Lanngalle ta siratangku

Masirik-sirik langgaukang parek kodi

Dasi na dasi

Na kubassukang

Minasanna tutowaku

Tekne ri lino

Kanang bone suruga

Makassar, 3 April 


\section{(7) Karaeng Malompoa}

Kaddejji kuasseng kuitung

Ajjalak kamateangku

Aknganro injak ri Karaeng Malompoa

O... Karaengku

Kilabbuiangak umurukku

Kukkulle akgauk bajik

Kaddejji kuisseng kuitung

Allo kalabbusangku

Aknganro injak

ri Karaeng Matinggia

O ... Karaengku

Teaki bubbuki nyawaku

Rioloang ta tobakku

Kaddejji kuasseng

Malaekak lambuntuliak

Kupalaki kupakalannying kalengku

Takmuri ambokoi lino

Rannu battu nibuntuli

I nakkeminne rupa tau

Mallak ri passessana naraka

Kipammopporangi dosaku 
Kisareak pappinyamang siratanga ri nakke

12 Juni 2016 


\section{(8) Ammakku Anrong Kalengku}

Ammakku anrong kelengku

Anrong tumallassukangku

Lompo panngai

Malompo pammaling-maling

Ri kambe anak-anakna

Battu bosi kabosiang

Battu bambang kabambangang

Tuli battui ri nakke

Manngerang tekne

Mammolik panngai

Mapparampe parek bajik

Ammakku anrong kalengku

Tutenayya sanrapanna

Buluk matinggi naambik

Bombang manggalluruk nasombalang

Parang tattarak nalalanngi

Majjappa bangkeng

Ta napisakringi posoa

Lanri manngaina ri anakna

Manna kajeknekang bangkenna 
Akkacannong palak limanna

Tuli tonji napattuju

Lanri erokna

Nacinik anakna tekne

Dasi na dasi

Kukkulle akgauk bajik

$\mathrm{Ri}$ anrong tumallassukangku

Tenayya ranggaselana

Mattaranak, mappakalompo

ri anak jari tauna

Makassar, 2013 


\section{(9) Kuparabbami Ulungku}

Kuparabbami ulungku

Ri pallungang kanrupikku

Kurakak boddong

Anrong tumallassukangku

Nasissik napakrupamang

Sakgenna tasselak matangku

Matangku akkaca-kaca

Tassungekngek ri ampikna

Kumanngukrangi

Kadosangku ri ammaku

Ammakku punna kurakak

Tuli mappasang ri nakke

Kuteak lalo

Takkaluppa ri kalengku

Ta manngukkrangi

Ri Karaeng Malompoa

Kalengku manna mabella

Ri tutowa malabbirikku

Ta lebbak kupisakringi

Ka panngainna 
Tuli makkaluk ri nakke

O... Karaeng Malompoa

Kisareak empoang masunggu

Tallasak empo matekne

Kukkulle todong

Appasabbi parek bajik

Ri parangku rupa tau

Ri bija pammanakangku

Labbipa ia ri ruwa tunnganakkangku

Makassar 2013 
(10) Anrong Manggeku

Anrong manggeku

I kattemintu tutowa malabbirikku

Lompo panngai

malompo panngamaseang

Manna bosi manna ribuk

Manna battu sassang makkapuk

Tuli tonjaki attuju

Solla na niak

Dallek hallalak kitayang

Ammakku, manggeku

Manna kajeknekang bangkenta

Tuli tonji kipajappa

Manna kacannong limanta

Tuli tonji kipattuju

Tena pariksik kisakring

Tena battalak kisongong

Tena sukkarak kigappa

Lanri rellata

Annarima panrannuang

Ri anak jari tauta 
Anrong manggeku

Lanri lannyinna nyawata

I katte kuparek picuru

Lanri bajikna ampeta

I katte kuparek guru

Lanri adelekna bicaranta

I katte kuparek panngulu

Lanri lambusukna oloanta

Anrong manggeku

Kipammopporangi dosaku

Kipappalak doangangangak

Barang kugappaji pakminasanta

Kukkulle ampaempoki ri tekne

I katte ruwa tau towaku

10 Mei 2013 


\section{(11). Kurapang Bulaeng Tiknok}

Nampaki i lalang battang

Kukimbolong allo banngi

Kumakminasa allassukang

Anak bulaeng tiknok

Nikarannuang ri anronta ri manggeta

Ri bija pammanakanta

Kurapangki bulaeng tiknok

Bulaeng tena ugianna

Tena namminra manna tattunggeng linoa

Tuli maccaya manna takgiling alloa

I kattemintu, anak

Kuminasa annyingarri tumasassang

Appakatekne tau malabba

Appakaingak ri tau takkaluppayya

Manna mabella lampanta

Tuli eranji adatta

Pappasanna tutowata

Teaki makring napaklingu tumasala

na jinak tukalumannyang

Teaki lomo nierang ri kajekkongang

I kattemintu 
Anak tunirannuangku

Anak bulaeng tiknok

Matantang kana ri katojengang

Malompo sirik na pacce

Mamallak makgauk kodi

Tanjengki ri Alla Taala

Kisalamak ri jappanta

I katte bajik, tutowata bajik todong

Dasi na dasi kikamma bulaeng tiknok

Tuli ningai

Tuli nikarannuang

Manna kere-kere mae

Makassar, 3 April 2018 


\section{(12) Kereji Janjinta}

Kereji antu janjinta, Daeng

Ri wattunta abbayuang

Ia kananta

Erokak kiparek tope

Ta malakkak ri kalenta

Sipabangung sipatinro

Singai sikamaseang

Kananta mae ri nakke, Daeng

Erokki sipassiriki, sipappaccei

Makrurung kana

Manggaukang parek bajik

Ri tanngana tujaiyya

Anne kamma, Daeng

Kikaluppaimi antu

janji lebbaka kipotok

I katte ammotok

$\mathrm{Na}$ i katte mallakgasai

Tena kumminra

Namaraemmo giotta

Panngainta kamma todong 
Bajikna na niak imangku, Daeng

Kulamung ri barambangku

Kaddekka kapang

Sallomak nikaluppai

Lanri gautta

Ammakrisi tamattappuk

O ... Daeng

Sekreji kupalak ri dallekang malabbiritta

Kiukrangi janjinnta

Kitobakkangi dosata

Kippalak panngamaseang

ri Karaeng Malompoa

Dasi na dasi

Na nipagiok nyawata

Ammoterek kamma ri memanganna

I katte manngai,

Nakke manngarimananngi

Nasabak Karaeng Allahu Taala

Makassar, 27 Mei 2016 


\section{(13) Manggeku}

Mangge teaki pallakki, kodong

$\mathrm{Ri}$ anak jari tauta

Kingai tongak

Sangkamma parangku anak

Manna empoak kamase, Tetta

Tuli sannanji nyawaku

Tuli sunggu tallasakku

Punna tuliki manngai,

mammaling-maling

manngamaseang ri anak jari tauta

Sungguna taua kucinik, Tetta

Nirurungang ri manggena

Niremba-remba

$\mathrm{Ri}$ anrong tullassukanna

I kambe anak jari tauta

Cinna nirurungang ri mangge

ni remba-remba ri anrong tullassukangku

Manggeku,

Teaki makring najinak, kodong 
Pannyonyokna Ibilisik

Tenaja antu

Nanngerang ri kabajikang

Labbipa ri anak jari tauta

Kabajikanna ammakku

Tuli rerang kiukrangi

Kiparek pau

Kiparek paruntuk kana

Tekneintu pakmaikna

Anak tunijarianta

Manna cella nikaddokang

Punna nacinik

Anrong manggena

singai sikamaseang

sipabangung sipatinro

Makassar, 28 Mei 2016 


\section{(14) Kaddek Assikola Tonjak}

Kaddek assikola tonjak, kodong

Ri wattungku caddi-caddi

Tenaja kapang

Na kamma anne sarengku

Sarengku anne wattua, gallek

Tena memampa namminra

Sabak taena kuppilanngeri

Taena kuerok asisikola

Ri wattungku caddi-caddi

Sikolapantu niondang

Panngissengang niballaki

Nampa akkulle

Tappainra tallasatta

Solla i nakke nabattui sassalalang

Tallasaknanjo agangku

Teknei na salewangang

Sabak erokna appilanngeri

Assikola ri caddina

Sassaklalammak i nakke, kodong 
Tallasak takronang-ronang

Lanri tenana

Kussikola ri caddiku

Taena todong panngisengang kuballaki

Kupasangi jari-jariku

Kuparek pappakaingak

Teako nakke, anak

Pituruki ri caddiku

Barang i kau

Nakkulle minra sarenta

Makassar, 2 April 2018 


\section{(15) Kaddek Tena Kussikola}

Kaddek tena kussikola, ammak

Ri wattungku caddi-caddi

Tulimak kapang

Tallasak kamase-mase

Nipajere kairi kanang

Nasuro-suro taua

Kamase-mase manggeku

Lanri tena sikolanna

Tuna ammakku

Ka tena panngissenganna

Barang anakna tena tonja anturuki

Panngisenganna taena

Barang-barang tena todong

Manna na kamma

Erojji appasikola

Barang anakna ampaempoi ri tekne

Napasikolak manggeku

Ri nampangku caddi-caddi

Barang i nakke

Nakkulle minra sarenna 
Sarenna tau towaku

Tena nakkulle amminra

Kadde taena

Panngissengang kuballaki

Kuballakinnami anne

Panngissengang kuboyayya

Nakkulle tommo

Pilak bajik tallasakku

Kupaempo tutowaku

Ri empoang mateknea

Kakkulle tommak

sanrupa parangku tau

Makassar, 2 April 2018 


\section{(16) Teaki Makring Najinak}

Teaki makring najinak

Tau lompo caritanna

Gassingka antu

Ase ngowarak rapponna

Nampa sannak bulerekn

Rapponna anjo asenna

Sannak aseng bulerekna

Mingka taena makring

Nipatimbo lamunganna

Taena bajik manngerang rappo

Lamungang nupatimboa

Allei pilei bajik

Taena angkana bulereknaji nucinik

Na mammattik iloroknu

Erok nuparek lamungang

ri tana balannu

Teaki patappak dudu

Ri tulompo bicarayya

Bajik mappau

Na kipinawang erokna 
Gassingi antu ri dallekantaji bajik

Bajikannganji tutua

Ala nabeta taua

Inai pasayu

Gassing nibeta panyonyok

Iaminjo kiparallu massing tutu

Kitea lomo turu-turukang

Ri tau takkullea lanngerang ri kabajikang

10 April 2018 


\section{(17) Pikkiri la Kikananga}

Ruwai tolinta tappampang

Bajik aseng takkaranna

Teaki makring

Sambarang kiparitoli

Punna niak tubbicara

Punna niak tubbicara

Tannang bajiki tolinta

Na kipinaknak

Kana bajikna taua

Kikasukmang bicaranna

Nakkulle amatu-matu

Punna niak la kipau

Pikkiri la kikananga

Apa erokna bukuang kananta

Solla nakkulle

Naaseng tuppinaknatta

Nakkulle akmatu-matu

Sukkuki antu bajikna

Punna bajiki taua

Lanri kananta

Naparek sikkok majarrek 
Nakkulle todong

Napakabajik sarenna

20 Juni 2018

\section{(18) Maeki Sipitangarri}

I katte rupa taua

Sannak antu sukkurutta

Lanri jaina belata

Akkulle kipitangarri

Punna niak gauk la kigaukang

Punna niak tau tea

Anngalleang tangarak tau maraeng

Tena nakkulle

Salewangang tallasakna

Tena tau

Ta niak kakuranganna

Taena tau niak kalabbianna

Iami anjo kiparallu sipitangarri

Maeki sipitangari

I katte sipammmanakang

I katte sipak-aganngang

Punna niak kasukkarrang

Solla nakkulle 
Tassala kasukkarranta

Nipadongkok songong battallatta

Ringang kalenta

Assombalang tallasatta

Anne ri tompokna lino

Makassar, 2 April 2018 


\section{(19) Pacce ta Naebbak Lading}

Paccei anne nyawaku

Dinging-dinging pakmaikku

Sangkontumami

Lokok naebbaka lading

Tuli makmikik punna neberoi jeknek

Kaddek pacce naebbajja lading

Akkule inji kutanggong

Ka niballei

Lintajji pia lokokna

Liung bate ebbakna

Anne kamma paknyawangku

Tuli paccei kusakring

Lanri kananna

Annokdok sangkamma jarung

Nampa taena na kuasseng kasalangku

E .. . Saribbattang

Kisailei bokota

Kikatutui lilata

Antu lilayya taena bukunna

Na mallokoki punna taena kitutu 
Punna nyawayyantu lokok

Sukkaraki balleianna

I nakke tommo

Kipaccei paknyawangku

Solla najjari pappilajarang ri katte

Makassar, 3 April 2018 


\section{(20) Ladingku Tonji Kupake}

Ladingku kupake anngebbak

Berangku kupake annebbak

Pangkulukku kupapangkuluk ri kayu

Na jai tau

Anngirimata ri nakke

Punna kupaebbak ladingku,

Jai tau kodi nyawa

Accinik mae ri nakke

Lanri taranna

$\mathrm{Na}$ lintak lebbak jamangku

Anjo na tarang ladingku

Ka tuli kukantisikna

$\mathrm{Na}$ kupattuju

Situruk matu-matunna

Ka teak nakke attuju ta ri kulleku

Appatuju ta ri kullena taua

Teaki kodi nyawai

Kodi mata ri taua

Iaji bajik kigaukang ri taua

Pakabajiki nyawata 
Pakatekne pakmaitta

Na kikutaknang

Apa lanrinna nakkulle bajik jamanna

Nakkulle todong kipicuru tallasakna

Inai-naimo tau

Bajik pakmaik ri tau

Lomoi antu annarima panngissengang

Ringangi antu dalleka battu ri ia

Lintaki antu annulung taua ri ia

Dasi-dasi kijjari tau bajik pakmaik

4 April 2018 


\section{(21) Pakabajiki Jamanta}

Anne ri kamma-kammayya

Jaintu tau anjama

Mingka assalak anjama

Sabak teyai

Jamanna na pariati

Iaji na pariati

Tubbuttayya sipakgadang

Ajo sarona

Punna lebbaki jamanna

Bajik ta bajik

Assalak lebbak najama

Nagappamintu wasselek bate jamanna

Punna niak la kijama

Kipajappai ottatta

Situruk panngissenganta

Na kipikkiri kabajikanna jamanta

Punna bajiki jamanta

Na rannuangki taua

Sekre rewasa

I katte naboya jamanta

I katte niparek picuru 
Lanri bajikna

Jamang lebbaka kijama

5 April 2018 


\section{(22) Teaki Pacalla-calla}

I katte rupa taua

Sekreji kabattuanta

Sekre assalak kajarianta

Taena tau sukkuk kaniakkanna

Sikamma rupa taua

Niak kalabbianna

Niak todong kakuranganna

Punna niakki nisare kalabbiang

Teaki tinggilanggai

Teaki pacalla-calla

Kitea todong tampo ri paranta tau

Kalabbianta panrannuanji ri katte

Punna niak kakuranganta

Teaki pikkiri dudu

Ka niakjantu kalabbiang kiballaki

Sukkuri passare-Na Karaenta

Nakkulle sannang nyawata

Salewangang tallasatta

Punna niak tau accalla

Kitarimai mabajik

Kisabbarrang paccallana 
Tambungki ri Karaenta

Iapa sallang ampamari pakrisitta

$\mathrm{Na}$ Ia tompa ambalasaki

Tau pacalla-callayya

Tukodia ri paranna rupa tau

5 April 2018 


\section{(23) Kaddejji Kapang Kiasseng}

Kaddejji kapang kiasseng

Pallokokinta kanayya

Pammakrisinna lilayya

Teamakintu

Akkana jarung ri nakke

Anjo kananta ri nakke

Lantangi na mallokoki

Tena naccerak

Mingka tuli akmikikna

Anngerang pakrisik lalang

Manna nakamma

Karampajji pakrisikku

Na kuballei

Siagadang kasabbarrang

I nakke lokok lanri kananta

Nakke kunrareng

lanri pakrisik kuerang

I nakke anngarruk

Na i kau manngakkali

Bolikmi kamma 
Pammakrisinta ri nakke

Ka taena na kiasseng

Kaddeji kapang kiasseng

Battuang kana jarunta

Tenaja kapang kierok akkana jarung

Ka tau niak kulleta

mingka kurangi pacceta

O... Daeng

Punna kikulle kalenta

Teaki tajjorok-jorok

Teaki talliwak-liwak

Ri tutuna kamasea

Anne lino rapang pammari-mariang

Allo riboko tena ta nimangeinna

Maeki tobak na kimassing anngukrangi

Dasi na dasi kinggappa panngamaseang

26 Desember 2018 


\section{(24) Kisailei Bokota}

Punna susa pakmaitta, Andik

Teaki pakrisik dudu

Tenaja antu

Na tuli mapakrisitta

Anne lino, rapang padati

tena na jannang

Sabak tuli allolokna

Punna cinikki tususa, Andik

Teaki kakkali dudu

Tenaja antu

Na tuli susa nyawana

Sekrentu wattu

tekne tonji tallasakna

Nyawa niaka ri katte

Alle katutui bajik

Teaki makring

Anngakkali tau susa

Appaciddak tusalasa

Punna tekne pakmaitta

Sannang katallasanta 
Sailei bokota

Teaki takkaluppai

Sikamma kaniakkanta

Karaeng Allahu pata

E... Saribbattang

Maeki kiparatutu

Massing ingak ri kaleya

Sailei bokota

Kimanngukrangi

ri Karaeng Mappajjaria

26 Desember 2018 


\section{(25) Kucinitta Anngarrukku}

Pammuji ta mattappuk

Kupasabbi ri Karaeng Malompoa

Kupparampe salawak ri Nabbita

Tarima kasi majai dudu

Kupabattu ri tau malabbirikku

Tau ampateteak ri kabajikang

Anngerangak ri empoang mateknea

Kucinitta anngarrukku

Lanri tekne pakmaikku

Kipaempo ri tampak kajannangangku

Panngukrangingku ri katte

Tenang tassala ri kalengku

Lanri i katte

Namminra katallassangku

Sassang makkapuk

Ajjari singarak kabuyu-buyu

Kana-kananta ri nakke

Tuli rerang kuukrangi 
Pannulunta ri nakke

Tuli ajjari sabbi malompo

Ri kalaccukanna atinta

Annulung tau kamasae

Jeknek matangku

Jeknek mata lanri rannu

Pammatei panngukrangi

26 Desember 2018 


\section{(26) Kicinikku Anngarutta}

Anngapa nasikna kamma

Pakmaitta ri kalengku?

Kicinikku anngarrutta

Assolong jeknek matanta

Napakamma dinging-dinging

Dinging-dingingi nyawata

Punna kicinik tanjakku,

Muwa-muwa rupangku

Takkulle kisabbi

Lanri niak panngukrangi

Takkulle kikaluppai

Jai purina sangkammanta

I katte tonji kicinikku anngarrutta

Jai kamanakang sangkammangku

I nakke tonji kikarruki kicinikku 
Punna niakak kicinik

Kisabbarrammi nyawata

Erojjak ningai nikamaseang

Mingka teak nikarruki

Lanri tuna tallasakku

Kilompoi sumangakku

Pakatekne pakmaikku

Kujjari bulaeng tiknok

Tuli tekne pakmaikku

Nikarannuang empo tallasak ri lino

26 Desember 2018 


\section{(27) Erokki Tea}

Punna narapik watunna

Allo pasijanjianta

Erokki tea

Tenamontu ta kammanna

Tenamontu ta nimangeinna

Punna narapik rapikna

Takkulleamaki mere

Erokki tea

Tena ta nimangeinna

Ka narapikmi janjinta

Ammantammaki makkale-kale

Tena barang tena doek

Tena tau

Anngalleangki bokota

Tena bija tena pammanakang

Ampalimbangki ri oloanta

Punna narapik janjinta

I kattemami nitayanngi pallampata

Erokki tea 
Tena ta nioloinna Pakkebbuk nipatajayya

Barang-baranta bellami accinik-cinik

Gauk bajittamami Ta malakkak ri ampitta

Maeki akgauk bajik

Manggaukang passuroang

Malliliang pappisangka

Dasi na dasi

Kisigappa amalak bajitta

Punna narapik pakkiotta

26 Desember 2018 


\section{(28) Ningai ta Nijampangi}

Niak anak ningai ta nijampangi?

Niak tojeng antu tau

Manngai manngamaseang

Mingka tena nanjampangi

$\mathrm{Ri}$ anak jari tauna

Anak ...

Ke mae manggeta?

Ke mae ammatta?

Anngapa kimantang takronang-ronang

ri ballak kajannanganta?

Anngapa kisusa Natinriang barang-barang majaita?

Anngapa kittinro Ta mallipak ta mabbaju?

Anngapa kikkaddok Ta siratanga kikaddok?

Anngapa kinggaukang

panggaukang ta nikarellaiyya?

Ke mae anrong manggeta?

Antekamma panngainna?

Tena tutowa tanngai ri anakna 
Tena tutowa tanngarimananngi ri anakna

Punna maknassa tau towa

Maknassa anrong mangge tojeng-tojeng

Iajia niak anrong mangge pallak ri anakna

Tanjampangi ri anak tunilassukanna

Lanri ta sawe ta sampanna

Napakamma pattujuang

Anngodang doek, appatambung barang-barang

Nampa anakna anjorengang tanrinniang

Taena na najampangi

Taena pakrambuang pepekna

28 Desember 2018 
(29) Tekne ta Nigollai

Kucinikna takmuriku

Anjanjang muwa rupanta

Tanjak tamallajjuta

Nyawa ta mamminrata

Tuli matekne

Tuli kanang ciikanta

I katte tekne

Tekne ta nigollai

Teknena teknea

Upakna upaka

Lanri tenana

Kitappuk panrannuang

Attayang panngamaseang

Ri Karaeng Malompoa

O... Karaeng

Punna kucinik taua

Angkanyamei tekne-teknea

Kuakluki ilorokku

Kukminasa

Kisareak pakkasiak mabajik 
Tekne ta nigollaia

Kipabellak ri sipak tinggi langgayya

Ri parangku rupa tau

Kijokjokangak agang malambusuka

Oloang lanngeranga ri kabajikang

Dasi na dasi

Kukkulle tuli salamak

Tekne ri lino

Tekneanngang ri anja

Ri tampak kajannangangku

Makassar, 15 Maret 2019-03-15 


\section{(30) Janna ta Nisantanngi}

Jaiji tau majanna

Kajjalak samarapanta

I katte janna

Janna ta nisantanngi

Tena nallajju

Nikanre makmole-mole

Tekne janna kigappayya

Teyai janna sossorang

Teyai passare-sare

Passanngalinna

Tittik songotta

Saro pakkaresoanta

Dallek niaka ri katte

Tuli kikasukkurinna

Tena kilabo

Taena na kisibakuk

Battu dalletta

Tanjengi ri Karaenta

Karaeng Mappajjaria

I katte janna

Tena kittunggaleng janna 
Lanri erotta

Annulung paranta tau

Kisare tete

Nakkulle tekne majanna

Dasi na dasi

Kituli empo tekne majanna

Bajik ri lino

Labbipa battu mangeang

Makassar, 15 Maret 2019-03-15 


\section{(31) Late Bunga Ta Malate Cinikanna}

Sallomak anne abboya

Bulaeng ta mamminrayya

Erok kuparek belo-belo

Passikkok batang kale

Pannyaleori ati masimpung

Pannyingarri ri sassang makkapuk

Manna siapa sallona

Tuli tonji kudanggangi

Manna kere-kere mae

Tuli tonji kukusissing

Tampak kajannanganna

Bulaeng tamamminrayya

Lanri cinnaku

Sidongkokang allo banngi

Singai sikamaseang

Ri tampak kajannangangku

Iapa kujannang tinro

Tallenguk ri paccinikku

Punna kugappa

Bulaeng ta mamminrayya

Manna malate bunganna 
Jappok lekok kayunna

Ta malate cinikanna

Pilak kujanjang

Pilak lantang panngainna

I nakke manngai

Ia manngarimananngi

Lanri Allahu Taala

Makassar, 15 Maret 2019 


\section{(32) Jammeng ta Nikaluppai}

Niak tau jammeng

Jammeng tulusujji bawang

Taena nammolik panngukrangi ri tau

Lebbak nilammak

Tammak tommi bicaranna

Niak tau jammeng

Jammeng tuli niukrangi

Lanri jaina gauk bajik naparampe

Ri paranna rupa tau

Taua punna tubajik

Bajikna tuli nipau

Manna taena naerok tuli nipau

Mingka taua

Tuli antu manngukrangi

Ukrangi allo ri boko

Kituli allamung bajik

Bajik nilamung

Bajik sallang nitaroi

Tumallinoa

Tuli narampe bajik lebbaka nilamung 
Niukranginna bajitta

Katambanna amalatta

Kamma lamung-lamung

Tuli orasak rapponna

Tena na talung manna tuli nitaroi

Bajik nilamung

Bajik narampe taua

Manna sallomaki jammeng

Makassar, 15 Maret 2020 


\section{(33) Bija Pammanakang}

Sallomak anne attayang

Tau nakkuk ri kalengku

Erok ningai

Erok nikarimananngi

Jaiji tubattu ri nakke

Mingka battuji paciddak

Lebbak attowak

Naputusammi Lampanna

O.. bija pammanakang

I nakkeminne bija kamaseta

Sallo attayang nakkuk

Erok ningai,

erok nikamaseang

Lanri Allahu Taala

Punna tanjappa kinakkuk

Barang-barampa kinngai

Panngissengampa kijalla

Tenamontu siratang

Sabak i nakke

Bija tuna kamaseta

Tanjak ta gakga 
Barang-barang ta maniak

Panngissengang bodo tompa

Bijangku,

Sekreji kupattowang

Manna tallasak kamase

Niak inja sirik pacce kuballaki

Tantang ri kontu tojeng

Ammenteng ri kalambusang

Tena kulari punna tojeng kupaenteng

Kumakminasa salewangang tallasakku

Makassar, 15 Maret 2019 


\section{(34) Teaki Lanre Mattayang}

I katte antu attayang

Niak inja tayanganna

Tau salloa kitayang

Mingka, anngapa katte

Kibboko ta massaile?

Punna posoki attayang

Anjallingki mae ri nakke

I nakke tayang

Tau tenamo ri lino

Manna nakamma

tuliji kukanakkuki

Mannanjo tena ri lino

Tuli tonji kutayanngi

Ka niak inja

Panngukrangi naparampe

Kumakminasa sibuntuluk battu mangeang

Teaki lanre mattayang 
Teaki bussang pakmaik

Punna kingai kitayanngi allo banngi

Na kiboya rurunganna

Tambungki ri Alla Taala

Kitea tappuk panrannuang

Battuji antu

Punna niak panngellai

Makassar, 16 Maret 2018 


\section{(35) Kupabellami ri Ati}

Nampaki siruppa mata

Sicinik ta sipattau

Kumakminasa

panngai sitojeng-sitojeng

Punna kucinik tanjatta

Kujanjang muwa rupanta

Ri kattemintu

Kulabuang panngaingku

Tusala cinijjak padeng

Manngai ta nijampangi

Kukana nakke

Tunngai tongki ri nakke

Sangkamma pakminasangku

Punna kammantu bateta

Anngerang sareak ri nakke

Kupabellami ri ati

Panngaingku ri kalenta

I nakke anne

Baine niak sirikku

Lompo panngalik 
Ri parangku rupa tau

Erammi anging mammirik

Panngai lebbaka tattiklak ri nyawata

Kimassing appalak dowang

Sibuntuluk julu tope

Narellaia Karaeng Allahu Taala

Makassar, 27 Maret 2019 
(36) Bokomak ri Panngainta

Punna niakmo kicinik

Bajikanngang na i nakke

Gakganngang na i nakke

Tekneanngang na i nakke

Rellamak antu

Kiboko ri panngainta

Panngaintantu ri nakke

Tuli tonji kuukrangi

Tena kumminra

Manna bokoki ri nakke

Kurapangki saribbattangku

Tuli mangai

Manngarimananngi ri nakke

Teaki pakrisik dudu

Ammolik simpung pakmaik

Punna kicinik tanjakku

Tabbialo ri dallekanna matanta

I nakke rapanjak pabbelo-belo

Kiparek pannyaleori

Kumakminasa tuliki kusaleori

Manna mabboko panngainta ri kalengku

Makassar, 25 Maret 2019 


\section{(37) Anakku}

Nampako kutiananngang

$\mathrm{Na}$ tena assipak kusakring

Apa kualluk

Kulanngeangi assuluk

Manna nakamma

Tuli tekneji nyawaku

Tuli rannu cinikangku

Lanri i kau sallo kuminasai

Anakku,

Salapang bulangko i lalang battang

Kuloserang allo banngi

Kukimbolong mange-mange

Manna nakamma

Tenaja kuppisakringi

Battalak erang battangku

Anakku,

Riwattunnu kulassukang

Tenamo pakrisik i rateanna

Manna nakamma

Tuli tonjak assumangak 
Sakgennu lassuk

Ri tompoknanne linoa

Teyai patok rannuku

Kulanngerekna sakrannu akngeak-ngeak

I kaumintu anak salloa kutayang

Lampaempoak ri tekne

Lassungkeangak bongongku

Lampiciniki singarak ri tutowanu

Makassar, 28 Maret 2019 


\section{(38) Pakminasa ri Anakku}

Anakku,

Puna towamak sallang

Sikalabbu-labbui manggenu

Teako sekkek

Punna niak nukanang

I kambe anne

Kana bajikmami kutayang

Teako gikgilik ri anrong manggenu

Tena antu na lebbak

Kurekeng kaposoangku

Anakku,

Karimananngi anrong manggenu

Katutui pammattikna jeknek matanna

Ka tena antu, Anak

Na kubiknyarak pappakalompoku

I kambe tau towanu

Teyai rante bulaeng kutayang

Nupakaluk ri kallongku

Teyai muttiara kukellai

Nupasang ri karemengku

Manna cella na pakkacci 
Kukaddokang allo banngi

Rannujak antu

punna tekne pakmaiknu

Anakku,

Inai-naimo anak

Doraka ri anrong manggena

Accinik tonjintu singarak,

mingka singarak kanjolik

Karimananngi anrong manggenu,

lanri Allahu Taala

Nummuntuluk singarak ta kalapakkang

Nasabak rella-Na Karaeng Allahu Taala

Makassar, 28 Maret 2019 


\section{(39) Kupalilimi Kalengku}

Teyai lanri tunaku

Teyai lanri kamaseku

Teyai tonji lanri kasiasiku

Na kupalili kalengku

Ri bija pammanakangku

Kupalili kalengku

Lanri sirikku

Tuli nikamaseang, nikodongang, nipakrikongang

Punna niakak nicinik

Alleok ri tujaiyya

Nicinikku nikarrukingku

Sanrapang anak makukang

Ta mak-anrong ta makmangge

$\mathrm{Na}$ i nakke tuniak tonja kulleku

Akkareso tojeng-tojeng

Kupalili kalengku

Ri tanngana bija pammanakangku

Ka erok tongi kupainra tallasakku

Bajik tompi tallasakku

Akkulle appakatekne

Kulleok ri bija pammanakangku 
Dasi na dasi

Kukkule nipacciniki

Makassar, 3 April 2019 


\section{(40) Kutetemminne Simpungku}

Kutetemminne simpungku

Kulilikang mange-mange

$\mathrm{Na}$ tena todong

Tumappattau ri nakke

Labbipa ia

Lanngamaseang ri nakke

Nakkenne anneteng simpung

Simpung battu ri taua

Tolongku simpung

Tenamo tunngamaseang

Passanngalinna

Tau pacciddajji niak

Kuasseng tommi kalengku

Nitallanngang ri likua

Tena salangku

Na i nakke tuli simpung

Lanri gaukna taua

Teak laloki eroki

Pasayu ri batang kalenta

Solla i nakke

Nisare simpung pakmaik 
Lanri taena na kututu ri kalengku

Makassar, 15 April 2019

\section{(41) Kulewai Nyawaku}

I nakke sanrapammamak

Biseang lebbak tallingge

Lanri lebbakna

Nipakalabba nyawaku

Nipakrisi pakmaikku

Nampa taena kasalangku lompo dudu

Manna nisareak kodi

Nibuanngi gauk ta kamma

Kulewaiji nyawaku

Kubajiki pakmaikku

$\mathrm{Na}$ kutarima passarena ri kalengku

I nakke anne kalengku

Narapangi barubbuk tau

Erok akkana, akanaji na sikamma

Eroki larro, larro tonji na sikamma

Sabak tuniak nakkulle abbakuk erok

I katte tau sunggua

Teaki talliwak-liwak 
Teaki tajjorok-jorok

Ri tukamase-masea

Sikamma kaniakkanta

Alla Taalaji pata

Makassar. 26 April 2019 


\section{(42) Kusingarri Paccinikku}

Kusingarri paccinikku

I pantarang pakrasangang

Ri borik tau sunggua

Ka erok tongak nipacciniki

Nipaccarammengi

Niparek picuru ri tanngana tujaiyya

Nampak lassuk ri linoa

Kubboya singarak lannying

Singarak ta kalapakkang

Lanri erokku assingarri empoangku

Kujjappa ri oloang malambusuka

Taena pammekanna

Taena todong

Katinting lannoddok bangkeng

Singarak malannying tuli kujanjang

Tuli kulio-lio

Dasi na dasi kusingarak satuli-tuli

Kunnyingarri tumasassang

Tallasak tuli matekne

Ri lino sakgenna anja

Lanri panngamaseang

ta mattappuk kutayanngi

Makassar, 28 Mei 2019 


\section{(43) Panngamaseanna Bijangku}

Panngamaseanna bijangku

Kutayanngi allo banngi

Kuniak rampe anrinni

Assombalangi kalengku

Lanri pasanna tutowa malabbirikku

Kaddeji tena nappasang

Tutowa malabbirikku

Tenamo kapang

Kusibuntuluk bijangku

Ia tanngasseng

I nakke tanngisseng tongi

Kusombalangi kalengku

Ri sekreang pakrasangang

Battuak mae

Attayangak panngamaseang

Lanri niakna kasiratanngang kuboya

Kuboyai anne bijangku

Lanri pasanna tettaku

Eroki bedeng

Sossoranna tutowana

Taena barang 
Manna sirijja na pacce

Makassar, 30 Mei 2019

83 


\section{(44) Kapeang Tommak}

Sallomak anne ammenteng

Ri dallekanna taua

Tena kukgiok, tena kukkana

Ka kammak turuntung sumangak

Accinik tau majai

Sumangakku anne kamma

Tenamo nasilalongang

Niak anraik, niak kalauk,

Niak timborang, niak warakkang

Attayang nikape akrappungang ri kalengku

Erokak appalak tulung

Ri katte tupanritayya

Nikapeang sumangakku

Lampa bellayya

Pakrimpungang ri kalengku

Kukkulle akkalepu

Anggaukang parek bajik

Ri parangku rupa tau

Anggaukang passuroang

Ri erokna agamaku

Makassar, 24 Desember 2019 


\section{(45) Ammattik Jeknek Matangku}

Kaddeji kuasseng memang

Tau la pallak ri nakke

Tau lammakrisi nyawa

Tau ta lammaling-maling

Tenaja kapang na kupinawang erotta

Erokna katte nyawata

Ampakrisi pakmaikku

Ampamattik jeknek matangku

Allokoki atingku

Kisare simpung tattappuk

$\mathrm{Na}$ tusannak kungainta

Ammattiki jeknek matangku

Punna kuruntuk kunawa-nawa

Pakmakrisinta ri nakke

Manna nakamma

Tuli tonji kuukrangi

Bajik lebbaka kilamung

I lalang ri pakmaikku

Na kulamungang

Pammakrisi lebbaka 
kipatimbo ri atingku

Barang kammai apa

Kijjari tau mabajik

Tanninriang sassallalang

Makassar, 24 Desember 2019-12-24 
(46) Niak Tonja Antu Sallang

Teaki lanre ri nakke

Punna niakak alleok

Ka erok tongak

Assalleorang tau bajik

Tau tekne pakmaikna

Niak tonja antu sallang

Na kiboyak ri pakmaik

Punna taenak

Kicinik ri dallekanta

Sabak i nakke tujai parutusangku

Ri gantingang gassinta

Bajikki sikanakkuki

Kittekne-tekne pakmaik

Kiparammolik panngukrangi

Ka umurutta takkulleai niboto

Saribbattangku,

Punna niak kana jule

Lebbak takrampe ri katte

Kuminasai i katte mappakakanang 
Na punna sala kanangku

I katte ampakanabai

Barang tuliki singai

Ri lino sakgenna anja

Makassar, 24 Desember 2019-12-24 


\section{(47) Siapaminne Salloku}

Siapaminne salloku

Ammempo sipattunggaleng

Ajjappa raik kalauk

Attinro ta mappaklungang

Annganre ta makkaddokang

$\mathrm{Na}$ tena todong

Tusikna mae ri nakke

Nakke anjo nipakamma

Ka tau tena bijangku

Mingka anakku

Tau anrinni pappokokanna manggena

$\mathrm{Na}$ tena kamma tau pacce ri anakku

Anakku tuli kupasang

Pakniak tongi siriknu na paccenu

Pajappai pikkirang bajiknu

Pakaresoi bukunnu

Nukkulle napacciniki bijannu

Solla i nakke ta nasaile bijannu

Punna bajik tallasaknu

Teako tonggilanggai 
Ciniki tau i rawanngannu

Nuccinik naik angkayaoi dallenu

Manna sikeddek punna hallalak na bajik

Iami antu maknassa barang-barannu

Makassar, 24 Desember 2019 


\title{
(48) Manna Jannammo Tinroku
}

\author{
Alle sai kiukrangi \\ Anne mae ri susata \\ Solla kitea \\ Nisarei takkaluppa
}

Anne mae ri susata

I nakke kipakrampei

Kiparek saribbattanta

Manna jannammo tinroku

Kupamillajji matangku

Na kuambangung

Assungkeiki pakkebbuk

Manna teyai wattu pakaddokang

Kupatalai kanreku

Kutowanaki kaddok

Lanri siknaku ancinik katallasanta

Anngapa katte naerok amminra nyawata?

Nakke anne appaingak

Lanri lebbakki sijanji

Inai-naimo sallang

Nabattui takkaluppa 
Ia parallu nisare pappakaingak

O... Saribbattangku

Teaki takkaluppai

punna anggapaki tekne

Ka anne lino

Tenaja na maknanuang

Makassar, 24 Desember 2019 


\section{(49) Carammengi Kalenta}

Carammeng labbak kitannang

Kiparek pabbakuk erotta

Solla kitea

Eroki abbakuk erok

Punna niak la kipau

Ri paranta rupa tau

Alle pikkiri

Ri gantig ta kipauna

Punna niak la kicalla

Ri paranta rupa tau

Carammengi kalenta

Gassing niajja callanta

Punna niak kikacinnai

Allei bakuk ri pakkulleta

Solla kitea kainranngang

Ri taua

Punna pasayuki antu

Lomo tongki amminawang

Apa kicinik erok asengi kialle

Manna tena kisiratang

Carammengi kalenta 
Kituli ingak

Kituli tutu

Sikontu panggaukanta

Makassar, 24 Desember 2019 


\section{(50) Sipakatau}

Kipissengi kalenta

Kusissing kabattuanta

Kitea tampo

Ri paranta rupa tau

Kitea takkaluppa

Ri Karaeng Mappajjaria

Punna kiasseng kalenta

Anngisengki appakatau

Appakakanang ri julu empota

Appakalabbirik ri tutowa labbiritta

Anngasi ri Daeng tuningaita

Sipanngalikiki simpangkatta

Anngamaseang ri anditta

Kituli mallak ri Karaeng Mappajjaria

Maeki sipakatau

I katte rupa taua

Sipakatuapakintu

Kikkalepu rupa tau

Rupa taua

Iapa na tau sitojennayya

Punna naaseng kalenna 
Naasseng tau maraenga

$\mathrm{Na}$ asseng Karaeng Mappajjaria

Napaempoi taua ri siratanna

Nagaukangi parentana agamana

E... Saribbattang

Maeki sipakatau

Dasi na dasi kinggappa panngamaseang

Kasalamakkang

Bajik ri lino labbipa sallang ri anja

Mei 2020 


\section{(51) Teaki Takkaluppai}

Sarenna rupa taua

Tena nassangkamma-kamma

Jai tusunggu

Jai tutuna na salasa

Jai tukasiasi

Jai tukalumannyang kalupepeang

Punna niasareki dallek

Teaki tajjorok-jorok bicaranta

Teaki talliwak-liwak giotta

Teaki takkaluppai ri assalak kajarianta

Lassuk ri lino ta manngerang apa-apa

Takkaluppakintu kapang

Punna kilantik kalenta

Lanri kaniakkanta

Tampo ri tau kamasea

Lanri pakkalumannyanganta

Tinggi langga ri tau samaraka

Lanri pangkatta

Anngukrangiki Saribbattang

Anne lino tenaja na maknannuang 
Rapanji pammari-mariang

Bajik kilamung

Bajik todong kitaroi

Kodi kilamung

Kodi sallang kitaroi

Bajik makodi i katte tonji pattantu

Mei 2020 


\section{(52) Kilambusi Paknyawanta}

Teaki makring najinak

Passawallang jai dudu

Manna sikeddek

Punna tenaja kijekkong

Ri paranta rupa tau

Punna niak kigaukang

Kilambusi paknyawanta

Teaki kossang

Teaki ranggaselai

Solla dalletta lancuk battu ri katte

Paknyawanta kilambusi

Kite ranggaselai

Apa gautta niajjantu barakkakna

Punna maknassa nyawata kipabajik

Kinggaukang parek bajik

Manna jai sawalatta

Wasselek kaposoanta

Tumbanji antu punna jekkong kipajappa

Na tena todong kinggappa kasalewangang 
Kasannanngang kamma todong

Kilambusi panyawanta

Ala apa kigaukang

Nampa kitayang

Kasselek kaposoanta

Nasabak Karaeng Allahu Taala

Mei 2020 


\section{(53) Curak Lipak Napilei}

Jaimintu lipak sabbe

Tattannang ri dallekanta

I kattemami

Ammile kikerokia

Kere curakna

Kanang ri bukkulenta

Curak lipak napilei

Kananga ri bukkulenna

Napasinturuk baju eroka napake

$\mathrm{Na}$ kamma inja nicallana ri taua

Apapinjo nicallangi

Curak lipak napakea

Sinturuk bunga bajunna

Kukana nakke tenamo la nicallangi

Manna kanang pakeanta

Punna kodi paknyawanta

Kodikero ri taua

Tenaja antu

Na kanang kacinikanta 
Curak lipatta

Lampa salai nicinik

Manna sallo kipilei

Iapa antu nabajik

cinikanna tummakkea

Punna kanang pakeanna

bajik mabajik ampe

Malumuk nyawa ri tau

10 Mei 2020

Kembong Daeng 
(54) Teaki Ranggaselai

Nakke tojemmak kamase

Empo tuna tallasakku

Mingka taena

Kuerok appassalasa

Ri parangku rupatau

Punna niak tau sare

Panrannuang ri kalengku

Teaki mallak

Teaki ranggaselai

Sabak i nakke

Tallasakkujintu tuna

Mingka sirikku

Sannak tonjantu lompona

Teaki ranggaselai

Abbata-bata ri nakke

Lebbak laloa

Tenapa kupassalasa

Kupallebbak jamangku

Kubassukang minasanta

Katte rannuang 
I nakke mappajjari

Dasi na dasi

Bajik ri katte

Mabbarakkak ri kalengku

Sabak laccukku

Mannarima panrannuang

Sabak rella-Na Allahu Taala

10 Mei 2020 
(55) Pakkeke Mappasisaklak

Nampaki uru sicinik

Sijanjang siruppa mata

Na tabbenrong tinakrakku

Nabattui songok dinging

Lanri mallakku

Manngai ta niruppai

Sukkuruk tena nattappuk

Mange ri Karaengku

Ka panngainta

Kontu golla na kaluku

I nakke manngai

I katte manngarimananngi

Punna sallang nirellai

Anjo pasingaianta

Katutui paknyawangku

Tarima kaniakkangku

I nakke rannuang ri katte

Anremba-rembak

Antama ri tampak.mateknea

Ri kajannanggang ta makkullea

nagesarak tau maraeng 
I nakke ri minasangku

Tallessokami cinikku

Tamminrayyami nyawaku

Erokak tuli singai

Tuli sikarimananngi

Sakgenta towa

Pakkeke tompa akkulle

Mappasisaklak

Dasi na dasi

Kisibuntuluk ri anja

Nipasiballak ri suruga

Kaminang teknea

Aamiin!

10 Mei 2020 
(56) Teaki Lanre Mattayang

Sannakmo antu upatta

Punna niakmo nitayang

Teaki lanre

Barang battuji ri katte

Tau salloa kitayang

Saloa kiminasai

Salloa kiantalai

Manna salloki attayang

Punna tantuja battuna

Tenaja antu

Kisia-sia attayang

Ka kamma tuttuju

Kigappaji kaposoanta

Teaki lanre mattayang

Antajai pabattuna

Na kiruppai

Panngai sitojeng-tojeng

Kitowana ampe bajik

Na kipaempo ri empoang mateknea

Punna battumo ri katte 
Tau salloa kitayang

Allei samara bajik

Golla nabinting

Kaluku kiparuppangi

Dasi na dasi

Tekne majanna kikaddok

I lalang katallassanta

Kituli bajik

Kituli sikatutui

Sakgenta towa

Tuli sunggu tallasatta

Tuli makanang cinikanta ri taua

10 Mei 2020 
(57) Tojengangi Kananta

Punna kikana kalenta

Tauki sitojeng-tojeng

Tojengangi kananta

Punna maknassa

Katojengang kipajappa

Lambusuk kipammentengi

I katte rupa taua

Kananta niparek sikkok

Apa kananta

Napatappaki taua

Iami anjo kiparallu

Ampikkiri kananta

Ri ganting ta kipasulukna

Punna antu tau bajik

Bajiki kana-kananna

Tena naerok

Sambarang la nakanang

Ri paranna rupa tau

Mallaki abballe-balle

Apa napau

Situruk panggaukanna 
Punna tojeng kipajappa

Kalambusang kipaenteng

Tantangki ri panggappata

Kitea makring

Naurikang tau sala

Lanngeranga kigauk ta mannabayya

Sikaliki abballe-balle

Manggaukang parek sala

Tulimi antu ta nipatappak kananta

Kanangi tojenga

Kimmenteng ri kalambusang

Tenamo antu na kisassalak kalenta

10 Mei 2020 


\section{(58) Danngalakminjo Ulunna}

Punna nicinik kalenna

Nikana tutena garrinna

Sannang nyawana

Salewangang tallasakna

Lanri jaina

Kabattuang panggappanna

Taua susa

Ia tuli mammakkalakna

Danngalakminjo ulunna

Tusarroa manngakkali

Sarroa patille-tille

Tena siapa sallona

Anngakkali tau susa

Nicinik tommi

Akkunrodong kamma jangang

Tuli kunrareng

Lanri danngalak ulunna

Napakamma pappikkirang

Susa tongi ta la garring

Ka tujai napikkiri 
Pattallassanna

Tenamo na silalongang

Nampa tujai panngerokanna

Iaminjo na nikana

Punna bajik tallasatta

Teaki takkaluppai

Niak tususa

Teaki kakkali dudu

Empoangantu

Tena natuli bajikna

Bangkenga cinik

Tena nappada ajjappa

10 Mei 2020 
(59) Jeknek Matangku

Sipariamami anne

Salloku kipakalabba

Kisare simpung pakmaik

Kipakanre paik

Mingka nampanna

ammattik jeknek matangku

Jeknek matangku nammattik

Teyai lanri gautta

Passanngalinna

Pannokdoknaji kananta

Ta kutanggong pakrisikna

Punna kicinikak sannang

Battuki mae ri nakke

Akkana jarung

Anngakkali tau simpung

kamma tutena

Risa-risa ri kalenta

Jeknek matangku

Sannak kukatutuinna

Punna ammattik 
Teai patok

Pakrisikna paknyawangku

I katte bija sungguku

Teaki talliwak-liwak

Bija tunata

Jagai jeknek matanna

Punna ammattik

Tayangi pabbalasak-Na

Karaeng Allahu Taala

10 Mei 2020

Kembong Daeng 


\section{(60) Rannungku ri Dosengku (Ibu Barbara Friberg/Timoty)}

Tabe, Kipammorammamak

Ri dallekang malabbiritta

Erokak anne

Ambacangki puisi bate limangku

Pammatei ranungku ri dosengku

Doseng Malabbirikku

Kuparampeangki kana pappasalamak

Lanri naiattamo limampulo taung

Singai sikamaseang, sikarimananngi

Siparutusuk allo banngi

Sipabangung sipatinro

Dasi na dasi

Kituli empo matekne

Empo salewangang

Ta naleok salibanra

Dosengku,

Nampaki uru kucinik

I katte ruwa sikalabine

Na kutantuangki tau malabbirik

Tau manngasseng 
Adelek ri bicaranta

Ri paranta rupa tau

Dosengku,

Kualleki picuru

Lanri kasomberanta,

Kalambusanta, bajik panngajaratta

Kalantanganna pangainta

Ri basa Bugis/Makassar

Lanri i katte

Allompoi sumangakku

Kussumangak amppapilajari basaku, sasateraku, na budayaku

Barang panngissenganta

Akkulleji na pakmatu-matu anak gurunta

O... Karaeng

Kilabbuiangi umurukna dosengku

Ia ruwa sikalabine

Kipabarakkak panngissenganna

Kitambaiang dallekna

Barang tuliji sileporang

Sanggena pakkeke mappasisaklak

Makassar, 18 Februari 2020 


\section{(61) Pappakalabbirikku ri Gurungku}

Gurungku

Ri wattunta uru kucinik

Kukanaki tau sangkang

Kukanaki tusibakuk

Kukanaki tukurang pammaling-maling

Ri kambe anak gurunta

Kukana ...

Ala siapa jaina panngarakgang tattiklak

$\mathrm{Ri}$ atingku

Ri tenanapa na kupinaknak sakranta

O... gurungku

Ri wattunta ammentenng ri dallekangku

Kujanjangki na tuli

makkutaknanang lalang nyawaku

I katte kijanjang tongak

sakge lompo panngamaseseng

Attakmuriki ri anak gurunta

Kisareak pallompoi sumangak

Kana na ampe mabajik

Kipasingarrangak pappilajarang

siagang sakra malompo 
Mabajik pabbattasakna

Kisareak pasaweang akkutaknang

Kisareak pasaweang sipitangarri

Kisareak panngarakgang siagang adelek

Tena kilebbak poso anngajarak

Mae ri kambe

I kattemintu tau malabbirik

Pammajikinta maknannuang kuukrangi

I katte gurungku

Gurungku picuruku

Gurungku antuntungak

Ri paknyawang malambusuka

Gurungku ampacinkangak

ampe mabajika

Gurungku anngajarak panngissengang

Guru ambimbingak ri jama-jamang mabajika

Sanggena kuasseng erokna

tallassaka na katallassanga

Gurungku tena kipajojjok.bawang

Tena kisamata assuro

Tena kinngalarroi, anggarattak

Mingka, kisareak picuru bajik ri ampeta, 
bukuang kananta na kedo-kedota bajitta

Tarima kasi guru

I katte picuruku

I katte pahalawangku

Pammajikinta tuli kupariati

Barang pakkusianta

Anggappaji amalak tattappuk

Tarima kasi guru

Kuparampeangki puisi

Tanra pappakalabbirikku

Makassar, 2 Mei 2020 


\section{(62) Ruwayya Tunnganakkangku}

Punna kuruntuk kunawa-nawa

Muawa-muwa rupanna

Panngainna, panngarimananginna

ruwayya tunnganakkangku

Teai patok nakkukku

Manna nakamma

Erokkumami niak

Ka tutowaku sallomi lingka ri anja

Kaddeji na niak inja

Ruwayya tunnganakkangku

Sipanakkukku sipamangeku ri ia

Amparampei kana bajik

Anggaukang passuroanna

Kupakatekne pakmaikna

Kupakasannang nyawana

Situruk pakminasanna

E ... Saribbattang

Kijampangi tutowata

Ri ganting atttallasakna

Teaki makring

Ampakrisi pakmaikna 
Punnantu jammeng

Tenamo na kisicinik

I katte nakkuk

Ri batetamami nakkuk

Dasi na dasi kisibuntuluk ri anja

Mei 2020 


\section{(63) Allerang Jeknek Matangku}

Punna kucinik agangku

Nirurungang, niremba-remba

$\mathrm{Ri}$ anrong manggena

Makgilingmamak

angkerek jeknek matangku

Napakamma dinging-dinging

Dinging-dingingi nyawaku

Allerang jeknek matangku

Ka nampak caddi

Nammaliang anrong manggeku

I nakkemami

tallasak takronang-ronang

Anjorengang tanrinniang

Tallasak empo salasa

Bajikna tonja na niak

Tau sikna ri tukukang

Manngamaseang

ri anak tau matuna

Mappakasunggu

ri anak tau salasa 
Kaddeka kapang

Tulimak takronang-takronang

O ... Karaengku

Kisareak sareak

Pakmaik mabajik

Tallasak empo matekne

Kukkulle akgauk bajik

ri tubajika ri nakke

Kurampe golla na kaluku

Kupakanre tekne na janna

Sanrapang anrong manggeku

Manna antu tumaraeng

Punna bajiki ri katte

Kukana tonja

Tutowa malabbirikku

Dasi na dasi

Pammajikinna

Anggappa amalak tattappuk

Nasabak ia

Kukkulle sanrupa parangku tau

Tallasak empo matekne

16 Mei 202 


\section{(64) Julu Borikku}

I katte julu borikku

Julu sampakrasangangku

Sekre tutowa nijulu

Sekre assalak kabattuanta

Sekre Karaeng Mappajjari

Kitallasak empo ri lino

Saribattang julu borikku

Teaki takkaluppai

Pasayu ri batangkalenta

Tabbesok ri gauk tamannabayya

Talliwak.jalla ri pabbarang-baranngang

Anne lino tenaja na maknannanuang

Narapik rapiitta nibokoi anne lino

Borik pammantanganta

Samborikku

Kupalaki pakmaik bajitta

Kierok ajjulu kana ri bicara kontutojeng

Ajjulu gauk ri panggaukang mabajik

Ajjulu paccinik ri kabajikanna tau tabballaka

Amminawangki ri pammarenta

Kimassare tangarak lanngeranga ri kabajikang 
Pakniaki siritta na pacceta i katte sipammanakang Julu borikku

Maeki sipakaingak punna niak takkaluppa

Sibosok ri kabajikang

Kitea sitindambiringi

I katte tau angkulleai kalenta

Kipatetei kabajikang tau tunayya

I katte tau tunayya akkasirikangki

Nakkulle tappainra tallasatta

Julu borikku

Maeki abbulo sibatang

Kippakabutti

panggauang mabajik

Ri borik malabbiritta

Dasi na dasi

Borik malabbiritta

Ajjari masunggu

Salewanganng tumabbuttana

Sabak rellana-Na Karaeng AllahuTaala

16 Mei 2020 


\section{(65) Singai Sikanakkuki}

Sannak antu kungainta

Sannak kukarimanganginta

I nakke manngai

I katte manngarimananngi

Kituli sikanakkuki

Iapantu na kinakkuk

Punna lantang panngainta

Iapa na kimanngai punna

Laccuk paknyawanta

Iapa kinnarimananngi

Punna bajiki ampe- ampeta

Iapanne niballaki kikkulke

Singai sikanakkuki

Situlung sikamaseang

E ... Saribbattang

Panngaingkuntu ri katte

Teaki ranggaselai

Nakke manngai nasabak

Allahu Taala

Punna niak kacallangku

Allemamak pacinikang 
Kipakanaba Lakkulle bajik kacinikangku

Saribbattangku

Punna nakkukak ri katte

$\mathrm{Na}$ taena pasaweang

Tenamo antu kijoja

Kitalipongak

Na kubaliki appau

Mannantu sakra

Kabayarrammi nakkukku

Labbipa ia

Punna kucinik tanjatta

Dasi na dasi

Kituli assingai

I katte sipammanakang

16 mei 2020 
(66) Tea Tonjak Nitunai

Manna tuna tallassakku

Empo kamase ri lino

Tena mangge tena ammak

Tena doek tena barang

Taena makring nipacciniki

Tea tonjak nitunai

Tallasakujinne tuna

Mingka niajja sirikku

Malompo tonja sumangak katallassangku

Erok sangkamma tusunggua tallasakna

Punna kicinik tanjakku

Kijanjang kaniakkangku

Teaki callai

Teaki appacidakki

Tenantu tau

Erok tuna na salasa

O ... Karaeng

Sallomak empo matuna

Alle tommak kamaseang

Kipatete ri oloanna mateknea 
Nakkulle todong

Amminra sarengku

Ajjari tau masunggu

Salewangang tallasakku

16 Mei 2020 